cal for superinfection by cytomegalovirus. Science. 2010;328(5974):102-106.

18. Slavuljica I, et al. Recombinant mouse cytomegalovirus expressing a ligand for the NKG2D receptor is attenuated and has improved vaccine properties. J Clin Invest. 2010;120(12):4532-4545.

19. Krmpotic A, et al. NK cell activation through the NKG2D ligand MULT- 1 is selectively prevented by the glycoprotein encoded by mouse cytomegalovirus gene m145. J Exp Med. 2005;201(2):211-220.

20. Lodoen MB, Abenes G, Umamoto S, Houchins JP, Liu F, Lanier LL. The cytomegalovirus $\mathrm{m} 155$ gene product subverts natural killer cell antiviral protec- tion by disruption of H60-NKG2D interactions. J Exp Med. 2004;200(8):1075-1081.

21. Pinto AK, Jamieson AM, Raulet DH, Hill AB. The role of NKG2D signaling in inhibition of cytotoxic T-lymphocyte lysis by the murine cytomegalovirus immunoevasin m152/gp40. J Virol. 2007; 81(22):12564-12571.

22. Arapovic J, Lenac Rovis T, Reddy AB, Krmpotic A, Jonjic S. Promiscuity of MCMV immunoevasin of NKG2D: m138/fcr-1 down-modulates RAE-1epsilon in addition to MULT-1 and H60. Mol Immunol. 2009;47(1):114-122.

23. Cicin-Sain L, et al. Targeted deletion of regions rich in immune-evasive genes from the cytomegalovirus genome as a novel vaccine strategy. J Virol. 2007; 81(24):13825-13834.

24. Crumpler MM, Choi KY, McVoy MA, Schleiss MR A live guinea pig cytomegalovirus vaccine deleted of three putative immune evasion genes is highly attenuated but remains immunogenic in a vaccine/ challenge model of congenital cytomegalovirus infection. Vaccine. 2009;27(31):4209-4218.

25. Söderberg-Nauclér C. Does cytomegalovirus play a causative role in the development of various inflammatory diseases and cancer? J Intern Med. 2006; 259(3):219-246.

\title{
Is ryanodine receptor phosphorylation key to the fight or flight response and heart failure?
}

Thomas Eschenhagen

Department of Experimental Pharmacology and Toxicology, Cardiovascular Research Center Hamburg, University Medical Center Hamburg Eppendorf, Hamburg, Germany.

\begin{abstract}
In situations of stress the heart beats faster and stronger. According to Marks and colleagues, this response is, to a large extent, the consequence of facilitated $\mathrm{Ca}^{2+}$ release from intracellular $\mathrm{Ca}^{2+}$ stores via ryanodine receptor 2 (RyR2), thought to be due to catecholamine-induced increases in RyR2 phosphorylation at serine 2808 (S2808). If catecholamine stimulation is sustained (for example, as occurs in heart failure), RyR2 becomes hyperphosphorylated and "leaky," leading to arrhythmias and other pathology. This "leaky RyR2 hypothesis" is highly controversial. In this issue of the JCI, Marks and colleagues report on two new mouse lines with mutations in S2808 that provide strong evidence supporting their theory. Moreover, the experiments revealed an influence of redox modifications of RyR2 that may account for some discrepancies in the field.
\end{abstract}

The heart has a remarkable capacity to react to altered demand by changing the rate at which it beats and the force with which it contracts, thereby changing its output. Both the reduction of cardiac output in phases of rest and its increase in physical and emotional exercise (the fight or flight response) are essential for normal body homeostasis and long-term survival. It is not surprising therefore that cardiac rate and force are regulated at multiple levels, extrinsic and intrinsic to the heart, and in a highly complex and secured fashion. Stimulation of $\beta_{1}$-adrenergic receptors by the sympathetic neurotransmitter norepinephrine induces increased production of the second messenger cAMP. cAMP directly and indirectly (via activation of PKA) induces faster depolarization in sinoatrial

Conflict of interest: The author has declared that no conflict of interest exists.

Citation for this article: J Clin Invest. 2010; 120(12):4197-4203. doi:10.1172/JCI45251. node cells (the cells that generate the action potentials that trigger cardiac contraction) and thus acceleration of heart rate (i.e., it has a "positive chronotropic effect") and stronger contraction (i.e., it has a "positive inotropic effect") and faster relaxation (i.e., it has a "positive lusitropic effect") in working myocytes. In chronic heart failure, one of the most frequent life-threatening diseases in Western societies, norepinephrine levels are chronically elevated, which leads to desensitization of the $\beta$-adrenergic signalling cascade and blunted responses. $\beta$-Blockers, introduced by Waagstein and colleagues in the mid 1970s, protect the heart from chronic sympathetic stimulation and provide the largest prognostic benefit for patients with chronic heart failure.

\section{Cardiac excitation-contraction coupling}

The positive inotropic and lusitropic consequences of $\beta_{1}$-adrenergic receptor stimulation in cardiomyocytes are explained by changes in excitation-contraction coupling, i.e., the relationship between the cardiac action potential and myofilament activation (Figure 1 of this commentary). When the cell depolarizes during a cardiac action potential, L-type $\mathrm{Ca}^{2+}$ channels (LTCCs) open, allowing $\mathrm{Ca}^{2+}$ to enter the cell. This so-called trigger $\mathrm{Ca}^{2+}$ induces a much larger $\mathrm{Ca}^{2+}$ release from intracellular $\mathrm{Ca}^{2+}$ stores, known as the sarcoplasmic reticulum (SR), via large tetrameric ryanodine-sensitive channels, referred to as ryanodine receptor 2 (RyR2). The increase in $\mathrm{Ca}^{2+}$ concentration initiates a conformational change in the myofilaments and thereby contraction. Removal of $\mathrm{Ca}^{2+}$ from the cytosol via the sarcoplasmic/endoplasmic reticulum $\mathrm{Ca}^{2+}$ ATPase (SERCA) and the $\mathrm{Na}^{+} / \mathrm{Ca}^{2+}$ exchanger (NCX) in the plasmalemma reverses the process. Importantly, the amount of $\mathrm{Ca}^{2+}$ entering the cell is, under normal conditions, exactly matched by the amount of $\mathrm{Ca}^{2+}$ leaving it via the NCX. $\beta_{1}$-Adrenergic receptors stimulate the system at numerous levels via PKA (Figure 1 of this commentary). Phosphorylation of LTCCs increases their open probability, allowing more $\mathrm{Ca}^{2+}$ to enter the cell. Phosphorylation of phospholamban (PLB), a small protein that when unphosphorylated inhibits SERCA, leads to disinhibition, i.e., increased reuptake of $\mathrm{Ca}^{2+}$ into the SR. This has at least two consequences: first, more $\mathrm{Ca}^{2+}$ in the SR and therefore more $\mathrm{Ca}^{2+}$ release during systole, which has a positive inotropic effect; and second, faster $\mathrm{Ca}^{2+}$ removal from the cytoplasm and thus faster 


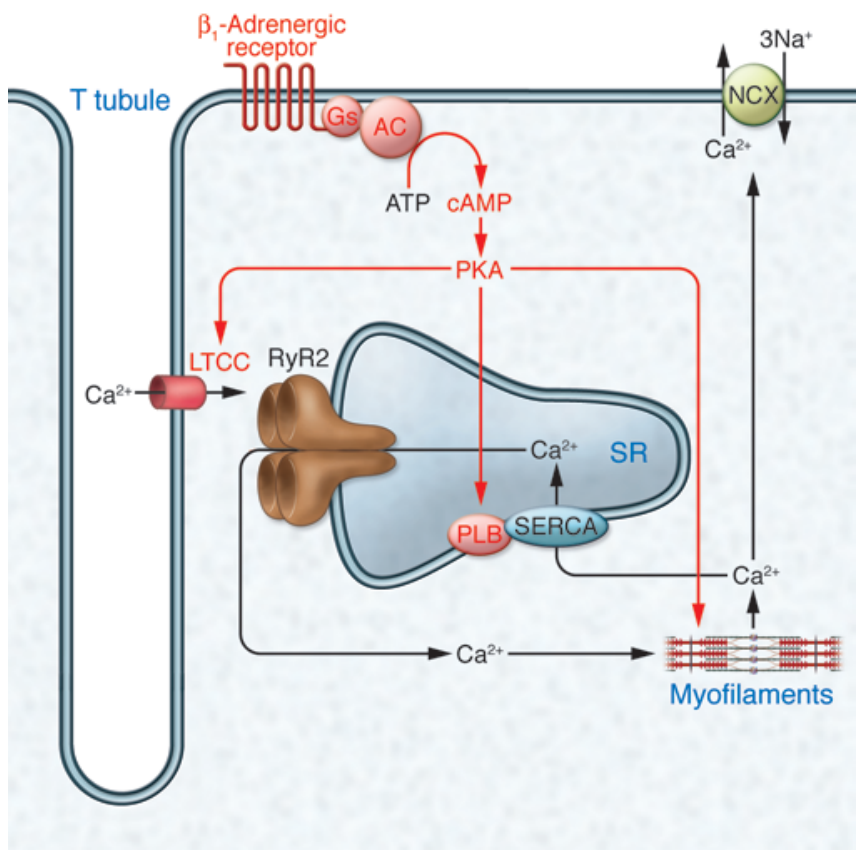

\section{Figure 1}

The classical view of cardiomyocyte excitation-contraction coupling and its regulation by $\beta$-adrenergic receptors. Under unstimulated conditions (black arrows), depolarization during an action potential opens LTCCs in T tubules, allowing $\mathrm{Ca}^{2+}$ to enter the cell. This trigger $\mathrm{Ca}^{2+}$ induces a larger $\mathrm{Ca}^{2+}$ release from the SR via RyR2. The increase in $\mathrm{Ca}^{2+}$ concentration initiates conformational changes of the myofilaments and thereby contraction. Removal of $\mathrm{Ca}^{2+}$ via SERCA and NCX reverses the process. Catecholamines stimulate excitationcontraction coupling (red symbols and lettering) by phosphorylating LTCCs (increased $\mathrm{Ca}^{2+}$ influx), PLB (increased $\mathrm{Ca}^{2+}$ reuptake into the $\mathrm{SR}$ ), and myofilament-based troponin I and myosin-binding protein $\mathrm{C}$ (increased relaxation). AC, adenylyl cyclase; Gs, stimulatory G protein. Adapted with permission from Nature (35). relaxation (i.e., a positive lusitropic effect). PKA also phosphorylates the myofilament proteins troponin inhibitor and myosinbinding protein $\mathrm{C}$, which desensitizes the myofilaments to $\mathrm{Ca}^{2+}$ and facilitates relaxation (i.e., positive lusitropic effects).

\section{RyR2 phosphorylation determines excitation-contraction coupling gain}

In the signalling scheme outlined in Figure 1 of this commentary, which prevailed until the end of the last century, the two major determinants of intracellular $\mathrm{Ca}^{2+}$ transients and thereby the contractile force of the heart were (a) the size of the $\mathrm{Ca}^{2+}$ current entering via the LTCC (well exemplified by the negative inotropic effects of LTCC blockers) and (b) the activity of SERCA and thus the $\mathrm{Ca}^{2+}$ load of the SR. The critical role of the latter was convincingly demonstrated by the fact that $\mathrm{Plb}^{-/}$ mice, which have maximal SERCA activity, exhibit higher basal force and reduced inotropic response to isoprenaline (1). In this scheme, RyR2 was just a passive gatekeeper, opening in response to the trigger $\mathrm{Ca}^{2+}$ and releasing $\mathrm{Ca}^{2+}$ at a quantity proportional to $\mathrm{SR} \mathrm{Ca}^{2+}$ concentrations. It is the merit of Marks and colleagues to have challenged that view with a whole series of papers (starting with their seminal paper in 2000; ref. 2), suggesting that PKA also increases the open probability of RyR2 by phosphorylation of serine 2808 (S2808) and the subsequent dissociation of FK506-binding protein 12.6 (FKBP12.6; also known as calstabin), a small regulatory protein that had been known since the early 1990s as a target of two immunosuppressant drugs, tacrolimus (also known as FK506) and rapamycin. Moreover, Marks and colleagues provided evidence that RyR2 is chronically hyperphosphorylated in human heart failure and therefore "leaky" (2). Leaky RyR2 channels in turn could explain both the reduced $\mathrm{Ca}^{2+}$ load of the SR (which leads to reduced contractile force of the failing heart) and the increased risk of spontaneous diastolic $\mathrm{Ca}^{2+}$ release, which, secondary to $\mathrm{Ca}^{2+}$ extrusion via the electrogenic NCX, could explain arrhythmias. In this theory, PKA phosphorylation of RyR2 and the subsequent dissociation of FKBP12.6 is the unifying mechanism underlying both the normal fight or flight response and heart failure.

\section{The controversy about the "leaky RyR2" hypothesis}

Appealing as Marks' theory is, the concept has been challenged and remains controversial (Tables 1 and 2). On the one hand, some theoretical considerations argue against it. For example, it seems counterintuitive that phosphorylation at a single residue in a protein of more than 5,000 amino acids could profoundly affect channel open probability. Second, S2808, the proposed site of phosphorylation by PKA, is located in an area distant from the FKBP12.6/RyR2 interaction site (3), making it somewhat unlikely that phosphory- lation affects FKPB12.6 binding. Third, it seems unlikely and to contradict experimental results (4) that an isolated increase in RyR2 open probability has more than a transient consequence on $\mathrm{Ca}^{2+}$ handling, because an isolated increase in $\mathrm{Ca}^{2+}$ release from the RyR2 will automatically lead to reduced $\mathrm{Ca}^{2+}$ load in the SR and therefore fast normalization of $\mathrm{Ca}^{2+}$ transients (autoregulation). Thus, leaky RyR2 channels alone, in contrast to super active SERCA (as in the case of Plb knockout; ref. 1), should not affect the basal force of contraction. Of course, theoretical arguments can be disputed and require experimental validation. It is now well established that point mutations in RyR2 can cause catecholaminergic polymorphic ventricular tachycardia (CPVT) - an electrophysiological disorder of the heart that can cause sudden death in young people as a result of arrhythmia - and hence, single-site phosphorylation could do so as well. In addition, the autoregulation argument does not necessarily hold if one accepts the idea that PKA phosphorylation of RyR2 is always accompanied by phosphorylation of LTCC and PLB and that RyR2 is just one out of (at least) three players in the concert.

More concerning than theoretical considerations are numerous reports that failed to reproduce important aspects of the data that support the leaky RyR2 hypothesis and the critical importance of S2808 (Tables 1 and 2). (a) Phosphorylation of RyR2 at S2808 has been found by others to 


\section{Table 1}

Overview of the controversy about the role of RyR2 phosphorylation in cardiac function under normal and disease conditions: hyperphosphorylation of RyR2 in heart failure and effect of $\beta$-adrenergic stimulation on FKBP12.6 binding and RyR2 open probability

\begin{tabular}{|c|c|c|c|c|c|}
\hline Result/interpretation & Method & Species & Group & Year & Ref. \\
\hline \multicolumn{6}{|l|}{ RyR2 is hyperphosphorylated in heart failure } \\
\hline $\begin{array}{l}\text { S2808-P increased in HF (approximately } \\
\text { increased } 800 \% \text { ) due to less PP1 } \\
\text { in RyR2 complex }\end{array}$ & IP and back phosphorylation & Human & Marks & 2000 & 2 \\
\hline No change in S2808-P & $\begin{array}{l}\text { Back phosphorylation, } \\
\text { phospho-specific Ab }\end{array}$ & Dog, human & Valdivia & 2002 & 6 \\
\hline No change in S2808-P, S2030 is major PKA site & $\begin{array}{c}\text { Phospho-specific Ab, } \\
\text { phospho-peptide mapping }\end{array}$ & Dog & Chen & 2005 & 8 \\
\hline $\begin{array}{l}\text { PP1 was up and FKBP12.6 was down in HF, } \\
\text { but there was less PP1 and FKBP12.6 in co-IP } \\
\text { with RyR2, more CaMKII in co-IP with RyR2, } \\
\text { and S2815-P increased } 65 \%-105 \% \text { and } \\
\text { S2808-P increased } 30 \%-63 \% \text { in HF }\end{array}$ & Homogenates and co-IP & Rabbit & $\begin{array}{l}\text { Pogwizd } \\
\text { and Bers }\end{array}$ & 2005 & 5 \\
\hline $\begin{array}{l}\text { S2808-P increased in LV (increased } 58 \% \text { ) } \\
\text { but not RV, S2808-P alone had no effect } \\
\text { on } \mathrm{Ca}^{2+} \text { leak }\end{array}$ & Post-MI HF & Rat & ter Keurs & 2006 & 7 \\
\hline \multicolumn{6}{|c|}{ ß-Adrenergic stimulation increases RyR2 opening via PKA-phosphorylation at S2808 and dissociation of FKBP12.6 } \\
\hline $\begin{array}{l}\text { PKA phosphorylation (and hyperphosphorylation } \\
\text { in HF) reduces FKBP12.6 binding and } \\
\text { increases Po }\end{array}$ & $\begin{array}{l}\text { Co-IP with or without PKA } \\
\text { and from NF vs. HF hearts }\end{array}$ & Dog, human & Marks & 2000 & 2 \\
\hline $\begin{array}{l}\text { S2809A mutant binds FKBP12.6 and has } \\
\text { identical properties as WT }\end{array}$ & $\begin{array}{l}\text { Recombinant expression } \\
\text { phospho-mutants }\end{array}$ & Rabbit & Meissner & 2003 & 12 \\
\hline $\begin{array}{l}\text { Dephosphorylation by PP1 or PP2a increases } \\
\mathrm{Ca}^{2+} \text { leak }\end{array}$ & $\begin{array}{l}\text { Permeabilized myocytes, } \\
\text { lipid bilayer }\end{array}$ & Rat, dog & Gyorke & 2003 & 11 \\
\hline $\begin{array}{l}\text { Phospho-mutations at } 2808 \text { or PKA phosphorylation } \\
\text { does not affect FKBP12.6 binding }\end{array}$ & $\begin{array}{l}\text { Recombinant mouse, } \\
\text { purified dog RyR2 }\end{array}$ & Mouse, dog & Chen & 2004 & 13 \\
\hline $\begin{array}{l}75 \% \text { basal S2808 phosphorylation, PKA and } \\
\text { dephosphorylation both increase Po }\end{array}$ & SR vesicles, lipid bilayer & Sheep & Sitsapesan & 2006 & 9 \\
\hline $\begin{array}{l}\text { Catalytic subunits of CaMKII and PKA both } \\
\text { increase RyR2 } \mathrm{Ca}^{2+} \text { leak, but PKA does so } \\
\text { only in WT, indicating exclusive effect on PLB }\end{array}$ & $\begin{array}{l}\text { Permeabilized WT and } \mathrm{Plb}^{-/} \\
\text {mouse myocytes with and } \\
\text { without inhibitors }\end{array}$ & Mouse & Bers & 2006 & 18 \\
\hline $\begin{array}{l}\text { S2808A mice display almost normal function } \\
\text { and response to Iso and are not protected } \\
\text { after TAC }\end{array}$ & S2808A mice & Mouse & Valdivia & 2007 & 28 \\
\hline $\begin{array}{l}\text { S2808 and S2030 are not located close to } \\
\text { FKBP12.6 binding site }\end{array}$ & $3 \mathrm{D}$ reconstruction of mouse RyR2 & Mouse & Chen & 2008 & 3 \\
\hline $\begin{array}{l}\text { Normal Iso response in vivo and } \\
\text { Langendorff-perfused hearts }\end{array}$ & S2808A mice (Valdivia) & Mouse & Houser & 2008 & 29 \\
\hline PKA and JTV 519 both reduce FKBP12.6 binding & Binding on purified RyR1/2 & Rabbit, pig & Lai & 2010 & 14 \\
\hline $\begin{array}{l}\text { The ratio of FKBP12/12.6 }=>10 \text {, most FKBP12. } 6 \\
\text { was bound, binding unaffected by PKA but } \\
\text { sensitive to rapamycin }\end{array}$ & $\begin{array}{c}\text { Permeabilized CM, } \\
\text { GFP-labeled FKBP12/12.6 }\end{array}$ & Rat, mouse & Bers & 2010 & 10 \\
\hline
\end{tabular}

CM, cardiomyocytes; HF, heart failure; JTV 519, experimental drug assumed to stabilize FKBP12.6 binding to RyR2; NF, nonfailing; Iso, isoprenaline; MI, myocardial infarction; PKA-P, phosphorylated PKA; PP1/PP2a, protein phosphatase 1/2a; Po, channel open probability; S2808-P, phosphorylated S2808; TAC, transaortic constriction inducing increased afterload.

be either not altered in heart failure at all or to be only moderately increased (5-8). Others have reported that $75 \%$ of the available RyR2 S2808 sites are phosphorylated under normal conditions, making a 9-fold change in chronic heart failure somewhat unlikely (9). (b) Whereas general consensus exists that $\beta$-adrenergic stimulation increases spontaneous $\mathrm{Ca}^{2+}$ release (the "Ca ${ }^{2+}$ leak") from the SR, the role of RyR2 phosphorylation and FKBP12.6 dissociation remains controversial. Importantly,
PKA had no effect on $\mathrm{Ca}^{2+}$ release in permeabilized $\mathrm{Plb}^{-/-}$mouse myocytes, i.e., cells in which the SR is maximally loaded with $\mathrm{Ca}^{2+}$ and one would have expected a particularly strong effect of increasing RyR2 open probability (10). These data suggest 


\section{commentaries}

\section{Table 2}

Overview of the controversy about the role of RyR2 phosphorylation in cardiac function under normal and disease conditions: FKBP12.6 binding in CPVT, effect of FKBP12.6 on RyR2 open probability, and the effect of oxidation and nitrosylation on RyR2 open probability and FKBP12.6 binding

Result/interpretation

Method

\section{Arrhythmogenic RyR2 mutations go along with reduced FKBP12.6 binding}

Fkbp12.6-- mice show exercise-induced arrhythmia, mutated RyR2 shows decreased FKBP12.6 binding and increased Po

Mutated RyR2 exhibits normal or slightly increased binding affinity for FKBP12.6, redox state affects binding

\section{FKBP12.6 inhibits Ca release from RyR2}

Rapamycin decreases FKBP12 binding and increases RyR2 activity

Removal and rebinding of FKBP12.6 to RyR2 without effect on activity

FKBP12.6 overexpression increases SR $\mathrm{Ca}^{2+}$ load and reduces $\mathrm{Ca}^{2+}$ sparks

Removal of FKBP12.6 has no effect on ryanodine binding, $\mathrm{Ca}^{2+}$ release, or arrhythmia, Fkbp 12.6-/- mice show no stress-induced arrhythmia

Transgenic FKBP12.6 overexpression reduces arrhythmia

Spark frequency was higher in Fkpb12.6 $6^{--}$ mice but they had normal Iso response

FKBP12.6 but not FKBP12 slightly inhibits $\mathrm{Ca}^{2+}$ release

Sticky FKBP12.6 reduces $\mathrm{Ca}^{2+}$ leak but does not rescue contractile or structural pathology of CaMKII TG

\section{Nitrosylation and oxidation affects RyR2}

NO inhibits RyR2 Po, SH-group reduction reverses this effect

Little oxidation had no effect, medium oxidation increases in $\mathrm{Po}$, strong oxidation inactivates

RyR2; no role of S-nitrosylation and tyrosine nitration loss of $\mathrm{SH}$ groups $+\mathrm{Ca}^{2+}$ leak

Nos1-1- mice: less RyR2 nitrosylation, more $\mathrm{SH}$ oxidation, increased $\mathrm{Po}$

GSNO but not NO directly affects RyR2 in a $\mathrm{pO}_{2}$-dependent manner

\section{Binding of FKBP12.6 to RyR2 is redox sensitive}

$\mathrm{H}_{2} \mathrm{O}_{2}$ and diamide reduce FKBP12.6 binding by $25 \%$ and $50 \%$, respectively, JTV 519 did not rescue

$\mathrm{SH}$ oxidation in $\mathrm{Nos}^{-/-}$mice was not associated with altered FKBP12.6
HF associated with (antioxidant-sensitive) binding to RyR2
Fkbp12.6-- mice, recombinant RyR2 with human mutations in lipid bilayer

Recombinant WT and mutated RyR2,

Co-IP and lipid bilayer
SR vesicles, lipid bilayer
Adenovirus overexpression
Coexpression HEK, lipid bilayer
Fkbp12.6-- mice
Conditional TG
Fkpb12.6--
GFP-labeled FKBP12/12.6
in permeabilized CM
FKBP12.6D37S TG $\times$ CaMKII TG

SR vesicles lipid bilayer, L-arginine, NOS inhibitors

SR vesicles, lipid bilayer, number of free $\mathrm{SH}$ groups

Pacing-induced HF with or without antioxidant, SR vesicles

Nos ${ }^{1-1-}$ mice, Nos3 $^{-1-}$ mice, or DKO

SR vesicles, lipid bilayer, different p02

SR vesicles, FKB-P12.6 binding with or without DTT, $\mathrm{H}_{2} \mathrm{O}_{2}$, diamide

$$
\text { Nos1-1- mice, } \mathrm{Nos}^{-1-} \text { mice, }
$$
or DKO

\section{Species}

Group

Year

Ref.

Mouse, human Marks $2003 \quad 16$

Human

Lai

2009

17

$\begin{array}{lccc}\text { Dog } & \text { Marks } & 1996 & 20 \\ \text { Dog } & \text { Fleischer } & 1996 & 15 \\ \text { Rabbit } & \begin{array}{c}\text { Smith and } \\ \text { Hasenfuss } \\ \text { Mouse }\end{array} & 2004 & 22 \\ & \text { Chen } & 2007 & 24 \\ \text { Mouse } & \text { Mercadier } & 2008 & 21 \\ \text { Mouse } & \text { Ji and Kotlikoff } & 2009 & 19 \\ & \text { Bers } & 2010 & 10 \\ \text { Rat, mouse } & \text { Maier } & 2010 & 23 \\ \text { Mouse } & & \end{array}$

DKO, double-knockout mice; DTT, dithiothreitol (reducing agent); TG, transgenic mice. 


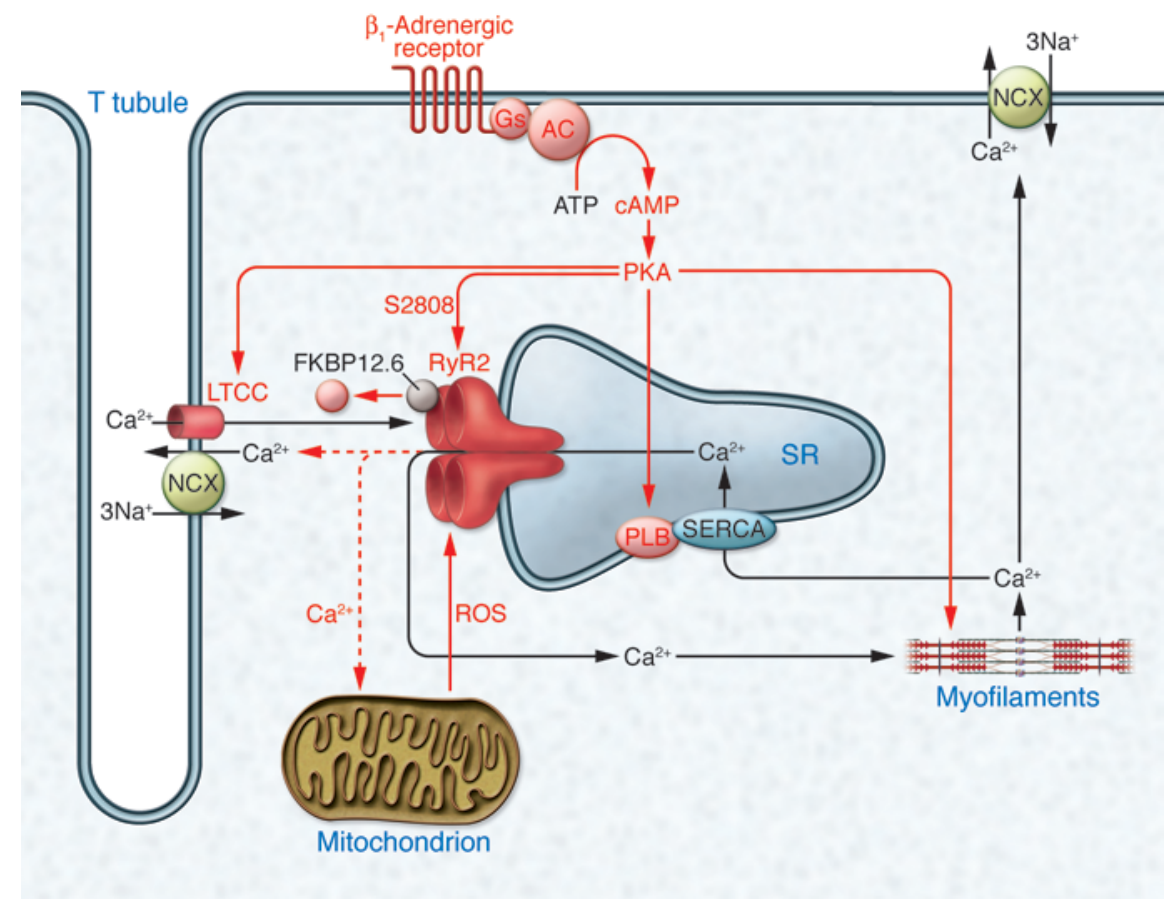

Figure 2

The leaky RyR2 hypothesis: phosphorylation and oxidation of RyR2 in the center of a vicious circle in heart failure. According to Marks and colleagues, catecholamines control excitationcontraction coupling gain not only at the level of LTCCs and PLB, but also at the level of RyR2 by phosphorylating it at $\mathrm{S} 2808$. The latter reduces RyR2 affinity for the stabilizing accessory protein, FKBP12.6, and increases its open probability. In heart failure, sustained catecholamine stimulation leads to hyperphosphorylation, leaky RyR2, spontaneous $\mathrm{Ca}^{2+}$ release and, via NCX, spontaneous depolarizations. The new data presented in this issue of the $\mathrm{JCl}(25,26)$ now suggest that phosphorylation at S2808 alone does not suffice to dissociate FKBP12.6 but that it needs oxidation of the channel plus phosphorylation. The level of oxidizing ROS is commonly increased in heart failure and, importantly, the increased $\mathrm{Ca}^{2+}$ leak from RyR2 (in consequence of oxidation and phosphorylation) further increases ROS production, e.g., from mitochondria. This constitutes a classical vicious circle. Black arrows and lettering indicate basic excitation-contraction coupling; red arrows indicate changes under chronic catecholamine stimulation and heart failure. The red dotted line indicates $\mathrm{Ca}^{2+}$ leak. Adapted with permission from Nature (35).

that the effect in WT myocytes is exclusively due to increased $\mathrm{SR} \mathrm{Ca}^{2+}$ loading (via PLB phosphorylation). This experiment also shows that the contribution of RyR cannot be judged without controlling for $\mathrm{SR} \mathrm{Ca}^{2+}$ load. Others have studied isolated RyR2 preparations and observed that not only phosphorylation at S2808, but also dephosphorylation, increased its open probability $(9,11)$. Several groups have failed to reproduce the effect observed by Marks and colleagues (2) of PKA phosphorylation at $\mathrm{S} 2808$ or mutations of this site on FKBP12.6 binding $(2,10,12,13)$, but others have (14). Moreover, a recent study by the Bers group (10) reported very low FKBP12.6 concentrations in rodent cardiomyocytes, resulting in only $10 \%-20 \%$ of RyR2 being occupied by FKBP12.6. These data, although in contrast to earlier data on isolated dog SR that reported more than $80 \%$ occupancy (15), suggest that a change of RyR2 affinity for FKBP12.6 would not affect more than a minority of RyR2. (c) Another exciting finding by the Marks group (16), namely that CPVT-associated mutations in RyR2 are associated with decreased FKBP12.6 binding, has also been challenged by reports of unaltered or even increased binding affinity of mutant RyR2 channels for FKBP12.6 (17). (d) Several reports support the idea that removal of FKBP12.6 from the RyR2 complex results in increased open probability, spark frequency, and arrhythmias $(18,19)$ and thus support this aspect of the Marks theory $(2,16,20)$. Conversely, overexpression of FKBP12.6 (or sticky mutants) was accompanied by a reduced propensity for cardiac arrhythmia (21-23). However, this did not improve cardiac pathology in a transgenic mouse model of heart failure (with increased $\mathrm{Ca}^{2+}$ leak), arguing against the universal role of leaky RyR2 in the pathophysiology of heart failure. Finally, others (24) could neither reproduce the effect of FKBP12.6 dissociation on RyR2 open probability nor the proarrhythmic phenotype of Fkbp12.6-knockout mice observed by the Marks group (16).

\section{Evidence from gene-targeted, phospho-mutant RyR2 mouse models}

In this confusing state of the RyR2 literature, one would expect "clean" knockin mouse models to finally provide a definite answer. Yet, this is only partially the case, as shown by two studies from the Marks laboratory published in this issue of the JCI $(25,26)$. The two papers report on two new mouse models: one in which the S2808 site was mutated to a non-phosphorylatable alanine (S2808A; ref. 25), and another in which S2808 was replaced by aspartic acid (S2808D), a "phosphomimetic" amino acid (26). The expectation was that S2808A mice would be less sensitive to the forceincreasing acute effects of isoprenaline (blunted fight or flight response) but also protected from adverse long-term consequences of $\beta$-adrenergic stimulation, such as cardiac dilatation and dysfunction (as seen in mice lacking phosphatase inhibitor-1; ref. 27). Conversely, S2808D mice would be expected to exhibit spontaneous FKBP12.6 depletion from the RyR2 complex, initial hypercontractility (because of facilitated $\mathrm{Ca}^{2+}$ release from the SR), spontaneous development of a cardiomyopathic phenotype, and exaggerated pathology under chronic stress. The results obtained by Marks and colleagues almost exactly matched these expectations and go beyond $(25,26)$. S2808A mice also exhibited blunted chronotropic responses (i.e., blunted acceleration of the heart rate) to isoprenaline and reduced exercise capacity, suggesting that RyR2 phosphorylation at S2808 regulates not only contractile force but heart rate too and that it is a critical determinant of physical fitness. The S2808D experiments also enlarge the picture by showing that the $\beta$-blockers metoprolol and carvedilol, while effective in infarcted WT mice, had no effect in S2808D mice. In contrast, the $\mathrm{Ca}^{2+}$ release channel-stabilizing drug S107 stabilized FKBP12.6 binding to RyR2 and ameliorated cardiac dysfunction in S2808D mice. Taken together, the 
new results from the Marks group strongly support a key role for phosphorylation of RyR2 S2808 in $\beta$-adrenergic regulation of cardiac force and frequency, in setting maximal exercise capacity, in determining cardiac responses to chronic stress, and in the protective effect of $\beta$-blockers.

\section{The new findings in the context of the published state of the art?}

Unfortunately, these new results from the Marks laboratory $(25,26)$ are in striking contrast to studies from the groups of Valdivia and Houser in an independently generated S2808A knockin mouse line $(28,29)$. These mice not only displayed normal heart dimensions before and after aortic banding but also normal myocyte shortening and $\mathrm{Ca}^{2+}$ transients, normal responses to isoprenaline, and, interestingly, normal PKA-catalyzed incorporation of radioactive phosphate into RyR2, despite effective elimination of S2808 phosphorylation. The latter result supports data suggesting that S2030 is the dominant PKA phosphorylation site on RyR2 and that S2808 is of minor quantitative relevance (8).

How can one reconcile these different results with apparently identical mutant mouse lines? Marks and colleagues present two major lines of reasoning $(25,26)$. The first one is that the other studies used doses/concentrations of isoprenaline and experimental conditions (pacing) that obscured differences in sensitivity. This explanation is based on a key experiment performed by the Marks group (25), showing that low doses of isoprenaline $(2 \mu \mathrm{g} / \mathrm{kg})$ had markedly smaller chronotropic and inotropic effects in S2808A than in WT mice but a 1,000-fold higher dose exerted similar maximal effects (Figure 1 of ref. 25). Indeed, the Valdivia and the Houser studies used saturating concentrations/ doses of isoprenaline in vitro $(1 \mu \mathrm{mol} / \mathrm{l})$ and in vivo $(2 \mathrm{mg} / \mathrm{kg})$, respectively $(28,29)$. However, dosing alone cannot account for the strikingly different observations for two reasons. First, the Houser group also tested the effects of isoprenaline on Langendorff-perfused mouse hearts at 10 $\mathrm{nmol} / \mathrm{l}$, a concentration that is clearly not saturating, and observed essentially identical effects in S2808A and WT hearts (29). Second, Figure 3 of ref. 25 shows, somewhat in contrast to their own findings presented in Figure 1 in the same paper, that the contractile force $(d P / d t)$ response was reduced in S2808A mouse hearts over the entire dose range (exhibiting a clear plateau at approximately $0.3 \mu \mathrm{g} / \mathrm{kg} / \mathrm{min}$ in both WT and S2808A mice). Moreover, a saturating concentration of isoprenaline $(100 \mathrm{nmol} / \mathrm{l})$ exerted less than a maximal force response in free-running Langendorff-perfused S2808A mice. Marks and colleagues therefore propose (25) that the differences between their results and those of the Houser group (at 10-fold lower concentrations) were due to the fact that Houser's group used paced Langendorff-hearts (480 beats/min). However, as demonstrated in Figure 3 of ref. 25, pacing (at 600 beats/min) reduced but did not abolish the differences between WT and S2808A mice. The Marks group also argues that in the Valdivia study (28) S2808A mouse myocytes did not react exactly as WT myocytes in response to isoprenaline (Figure 5C in ref. 28). Whereas this is formally true, the slightly (but statistically significantly) higher $\mathrm{Ca}^{2+}$ transient in WT myocytes at $3 \mathrm{~Hz}$ does not strike as a relevant difference to the author of this commentary. Finally, the Marks group shows in the second paper (26) that $\mathrm{Ca}^{2+}$ spark frequency in 6- to 8-month-old S2808D mouse myocytes was larger than in WT, despite lower SR $\mathrm{Ca}^{2+}$ load. It is difficult to explain these data by an isolated change in RyR2 open probability, because such situation automatically leads to a new equilibrium at a lower SR load (4).

A second hypothesis to explain the controversy relates to redox modifications of RyR2. It is well known and undisputed that RyR1 (the skeletal isoform) and RyR2 are redox responsive (Tables 1 and 2). RyR1 contains 101 thiols/subunit, and their oxidation (e.g., by ROS) increases RyR1 open probability at low/moderate levels of oxidation (and irreversibly inactivates it at high levels; ref. 30). RyR2 is also subject to oxidation and S-nitrosylation (likely via $\mathrm{S}$-nitrosoglutathione) in a $\mathrm{pO}_{2}$-dependent manner (31). Recent work suggests competition between NOS1/NO-dependent S-nitrosylation and ROS-dependent SH oxidation, with the latter leading to increased leakiness of the channel (32). One report indicates that $\mathrm{SH}$ oxidation reduces the affinity of RyR2 for FKBP12.6 (33), but another shows that the level of FKBP12.6 associated with RyR2 is normal despite its increased oxidation in $\mathrm{Nos}^{-1-}$ mice (32). In any case, there is little doubt that redox modifications can profoundly affect RyR2 function. Moreover, redox conditions can easily differ between dif- ferent protocols and laboratories and are rarely explicitly reported (e.g., some investigators use ascorbic acid in their Tyrode's solution, others do not). Thus, the idea that different redox states of RyR2 account for at least some of the published discrepancies is appealing. Based on experiments presented in Figure 1D of ref. 25, Marks and colleagues propose that oxidation of RyR2 sensitizes the channel for PKA phosphorylation at S2808 and destabilizes FKBP12.6 binding. Conversely, under non-oxidizing conditions, PKA may not induce FKBP12.6 dissociation, and this might then explain why others did not find it. The spontaneous cardiomyopathic phenotype of S2808D mice observed by Marks and colleagues was accompanied by increased S-nitrosylation and more pronounced $\mathrm{SH}$ oxidation (Figure 2B of ref. 26). This heart failure-associated increase in oxidation could then explain why it took 6 months for S2808D mice to exhibit significant loss of FKBP12.6 from the RyR2 complex (Figure 2, A and B, of ref. 26), despite the proposed "hyperphosphorylation state" of RyR2 being present throughout life. The hypothesis derived by Marks and colleagues from these results is that both oxidative stress (as commonly seen in heart failure; ref. 34) and PKA phosphorylation cooperate to destabilize the RyR2-FKBP12.6 complex (Figure 2 of this commentary). The increased $\mathrm{Ca}^{2+}$ leak then promotes ROS generation (e.g., from mitochondria) and thus accelerates the vicious circle (Figure 2 of this commentary). This elegant hypothesis could indeed explain some of the experimental discrepancies, particularly those in vitro. It is more difficult to assume that it can explain discrepancies between experiments in whole heart homogenates, intact cells/ hearts, and entire animals.

\section{Conclusion}

Although the controversies surrounding RyR2 phosphorylation are sure to continue, the means to directly test evolving hypotheses are at hand. Direct head-tohead comparisons of available animal models and other reagents in multiple laboratories and the further development of ever-more refined reagents will help to clarify this fundamental aspect of cardiovascular physiology.

\section{Acknowledgments}

The work of the author is supported by grants from the German Research Founda- 
tion (DFG FOR 604, Es 88/10-1), the European Union (EUGeneHeart, Angioscaff), and the German Heart Foundation.

Address correspondence to: T. Eschenhagen, Department of Experimental Pharmacology and Toxicology, Cardiovascular Research Center Hamburg, University Medical Center Hamburg Eppendorf, Martini-strasse 52, 20246 Hamburg, Germany. Phone: 49.40.74105.2180; Fax: 49.40.74105.4876; E-mail: t.eschenhagen@uke.de.

1. Luo W, et al. Targeted ablation of the phospholamban gene is associated with markedly enhanced myocardial contractility and loss of beta-agonist stimulation. Circ Res. 1994;75(3):401-409.

2. Marx SO, et al. PKA phosphorylation dissociates FKBP12.6 from the calcium release channel (ryanodine receptor): defective regulation in failing hearts. Cell. 2000;101(4):365-376.

3. Jones PP, et al. Localization of PKA phosphorylation site, $\operatorname{Ser}(2030)$, in the three-dimensional structure of cardiac ryanodine receptor. Biochem J. 2008; 410(2):261-270.

4. Venetucci LA, Trafford AW, Eisner DA. Increasing ryanodine receptor open probability alone does not produce arrhythmogenic calcium waves: threshold sarcoplasmic reticulum calcium content is required. Circ Res. 2007;100(1):105-111.

5. Ai X, Curran JW, Shannon TR, Bers DM, Pogwizd SM. Ca2+/calmodulin-dependent protein kinase modulates cardiac ryanodine receptor phosphorylation and sarcoplasmic reticulum $\mathrm{Ca} 2+$ leak in heart failure. Circ Res. 2005;97(12):1314-1322.

6. Jiang MT, Lokuta AJ, Farrell EF, Wolff MR, Haworth RA, Valdivia HH. Abnormal Ca2+ release, but normal ryanodine receptors, in canine and human heart failure. Circ Res. 2002;91(11):1015-1022.

7. Obayashi M, et al. Spontaneous diastolic contractions and phosphorylation of the cardiac ryanodine receptor at serine-2808 in congestive heart failure in rat. Cardiovasc Res. 2006;69(1):140-151.

8. Xiao B, et al. Characterization of a novel PKA phosphorylation site, serine-2030, reveals no PKA hyperphosphorylation of the cardiac ryanodine receptor in canine heart failure. Circ Res. 2005;96(8):847-855.

9. Carter S, Colyer J, Sitsapesan R. Maximum phosphorylation of the cardiac ryanodine receptor at serine- 2809 by protein kinase a produces unique modifications to channel gating and conductance not observed at lower levels of phosphorylation. Circ Res. 2006;98(12):1506-1513.
10. Guo T, et al. Kinetics of FKBP12.6 binding to ryanodine receptors in permeabilized cardiac myocytes and effects on Ca sparks. Circ Res. 2010; 106(11):1743-1752.

11. Terentyev D, Viatchenko-Karpinski S, Gyorke I, Terentyeva R, Gyorke S. Protein phosphatases decrease sarcoplasmic reticulum calcium content by stimulating calcium release in cardiac myocytes. JPhysiol. 2003;552(pt 1):109-118.

12. Stange M, Xu L, Balshaw D, Yamaguchi N, Meissner $\mathrm{G}$. Characterization of recombinant skeletal muscle (Ser-2843) and cardiac muscle (Ser-2809) ryanodine receptor phosphorylation mutants. J Biol Chem. 2003;278(51):51693-51702.

13. Xiao B, Sutherland C, Walsh MP, Chen SR. Protein kinase A phosphorylation at serine-2808 of the cardiac Ca2+-release channel (ryanodine receptor) does not dissociate 12.6-kDa FK506-binding protein (FKBP12.6). Circ Res. 2004;94(4):487-495.

14. Blayney LM, Jones JL, Griffiths J, Lai FA. A mechanism of ryanodine receptor modulation by FKBP12/12.6, protein kinase A, and K201. Cardiovasc Res. 2010;85(1):68-78.

15. Timerman AP, et al. Selective binding of FKBP12.6 by the cardiac ryanodine receptor. J Biol Chem. 1996;271(34):20385-20391.

16. Wehrens XH, et al. FKBP12.6 deficiency and defective calcium release channel (ryanodine receptor) function linked to exercise-induced sudden cardiac death. Cell. 2003;113(7):829-840.

17. Zissimopoulos S, Thomas NL, Jamaluddin WW, La FA. FKBP12.6 binding of ryanodine receptors carrying mutations associated with arrhythmogenic cardiac disease. Biochem J. 2009;419(2):273-278

18. Guo T, Zhang T, Mestril R, Bers DM. Ca2+/Calmodulin-dependent protein kinase II phosphorylation of ryanodine receptor does affect calcium sparks in mouse ventricular myocytes. Circ Res. 2006;99(4):398-406.

19. Zhang X, et al. Dissociation of FKBP12.6 from ryanodine receptor type 2 is regulated by cyclic ADPribose but not beta-adrenergic stimulation in mouse cardiomyocytes. Cardiovasc Res. 2009;84(2):253-262.

20. Kaftan E, Marks AR, Ehrlich BE. Effects of rapamycin on ryanodine receptor $/ \mathrm{Ca}(2+)$-release channels from cardiac muscle. Circ Res. 1996;78(6):990-997.

21. Gellen B, et al. Conditional FKBP12.6 overexpression in mouse cardiac myocytes prevents triggered ventricular tachycardia through specific alterations in excitation-contraction coupling. Circulation. 2008; 117(14):1778-1786

22. Loughrey CM, et al. Over-expression of FK506binding protein FKBP12.6 alters excitation-contraction coupling in adult rabbit cardiomyocytes. JPhysiol. 2004;556(pt 3):919-934.

23. Seidler T, et al. Limitations of FKBP12.6-directed treatment strategies for maladaptive cardiac remod- eling and heart failure [published online ahead of print August 24, 2010]. J Mol Cell Cardiol. doi: 10.1016/j.yjmcc.2010.08.016.

24. Xiao B, et al. Functional consequence of protein kinase A-dependent phosphorylation of the cardiac ryanodine receptor: sensitization of store overload-induced Ca2+ release. J Biol Chem. 2007; 282(41):30256-30264.

25. Shan J, et al. Phosphorylation of the ryanodine receptor mediates the cardiac fight or flight response in mice. J Clin Invest. 2010; 120(12):4388-4398

26 . Shan J, et al. Role of chronic ryanodine receptor phosphorylation in heart failure and $\beta$-adrenergic receptor blockade in mice. J Clin Invest. 2010; 120(12):4375-4387.

27. Wittkopper K, et al. Constitutively active phosphatase inhibitor- 1 improves cardiac contractility in young mice but is deleterious after catecholaminergic stress and with aging. J Clin Invest. 2010; 120(2):617-626.

28. Benkusky NA, et al. Intact beta-adrenergic response and unmodified progression toward heart failure in mice with genetic ablation of a major protein kinase A phosphorylation site in the cardiac ryanodine receptor. Circ Res. 2007;101(8):819-829.

29. MacDonnell SM, et al. Adrenergic regulation of cardiac contractility does not involve phosphorylation of the cardiac ryanodine receptor at serine 2808. Circ Res. 2008;102(8):e65-e72.

30. Sun J, Xu L, Eu JP, Stamler JS, Meissner G. Classes of thiols that influence the activity of the skeletal muscle calcium release channel. J Biol Chem. 2001;276(19):15625-15630.

31. Sun J, Yamaguchi N, Xu L, Eu JP, Stamler JS, Meissner $G$. Regulation of the cardiac muscle ryanodine receptor by $\mathrm{O}(2)$ tension and $\mathrm{S}$-nitrosoglutathione. Biochemistry. 2008;47(52):13985-13990.

32. Gonzalez DR, Beigi F, Treuer AV, Hare JM. Deficient ryanodine receptor S-nitrosylation increases sarcoplasmic reticulum calcium leak and arrhythmogenesis in cardiomyocytes. Proc Natl Acad Sci US A. 2007;104(51):20612-20617.

33. Zissimopoulos S, Docrat N, Lai FA. Redox sensitivity of the ryanodine receptor interaction with FK506-binding protein. J Biol Chem. 2007; 282(10):6976-6983.

34. Yano $\mathrm{M}$, et al. Correction of defective interdomain interaction within ryanodine receptor by antioxidant is a new therapeutic strategy against heart failure. Circulation. 2005;112(23):3633-3643.

35. Bers DM. Cardiac excitation-contraction coupling. Nature. 2002;415(6868):198-205.

36. Zahradnikova A, Minarovic I, Venema RC, Meszaros LG. Inactivation of the cardiac ryanodine receptor calcium release channel by nitric oxide. Cell Calcium. 1997;22(6):447-454. 